\title{
The Relationship between Cognitive-Attention Syndrome and Occupational Stress of Workers Exposed to Occupational Accidents
}

Zohreh Hashemi, ( ${ }^{\star}$ Corresponding author), Assistant Professor, Department of psychology, University of Maragheh, Maragheh, Iran. zhashemi1320@gmail.com

Taghi Shahjoee, M.Sc, Department of psychology, university of Tabriz, Tabriz, Iran.

\section{Abstract}

Background and aims: The present study has determined the relationship between the cognitive-attention syndrome and job stress of employees exposed to occupational accidents in Isfahan Steel Company..

Methods: The method used for the present study is cross-sectional and was performed on production line workers exposed to accidents in the steel production company in Isfahan in 2019. The statistical population of the study included all the workers of the Isfahan Steel Production Company. According to the size of the population, 234 workers were selected as a sample by simple random sampling, using Krejcie-Morgan table. The CAS-1 Cognitive- attention Syndrome Scale and the Steinmetz Job Stress Questionnaire were used to collect data. SPSS-18 software and Pearson correlation coefficient test and multiple regression analysis were used to analyze the data.

Results: Based on the research findings, there is a positive and significant correlation between the cognitive-attention syndrome and job stress ( $r$ $=0.094, \mathrm{p}<0.01)$. In addition, the results of regression analysis showed that the job stress has a significant role $(\mathrm{p}<0.01)$ in predicting employees' cognitive-attention syndrome. Regression coefficients showed that the job stress variable can significantly predict $88 \%$ of the variance of the cognitiveattention syndrome $(p<0.01)$. Regression coefficients show that the variable of job stress $(\mathrm{p}<0.01, \mathrm{t}=42.49)$ can significantly predict the variance of the cognitive-attention syndrome.

Conclusion: The Cognitive-attention syndrome is one of the factors affecting the level of job stress in employees that should be considered in the field of psychological well-being of employed people.

Conflicts of interest: None

Funding: None

\section{Keywords}

Cognitive-Attention

Syndrome

Occupational Stress

Employees

Received: 2019/11/02

Accepted : 2020/11/04 
Introduction

In addition to physical injuries, occupational accidents can have catastrophic effects on workers' psychological well-being. One of the major factors in the occurrence and persistence of mental disorders in people affected by an accident, is their exposure, content and mental state (1).

Occupational Stress is associated with many illnesses and workplace issues. This association has been studied and confirmed in cardiovascular disease, musculoskeletal disease, hypertension and some other diseases, but the relationship between occupational stress and occupational accidents is less discussed. In the metacognitive approach, a special style of thinking called cognitive-attention syndrome is discussed (2). Cognitive-attention syndrome includes, extreme conceptual processing in the form of anxiety, rumination, threat-focused attention and dysfunctional coping behaviors. In recent years, the study of thought patterns such as anxiety and rumination in emotional disorders, and the study of unwanted thoughts and its role in the persistence of emotional disorders has been considered by researchers. Ruminant, is described as a constant preoccupation of an idea or subject. Rumination seems to elevate the anxiety level and cause depression, and is associated with poor quality of sleep, sleep disturbance and suicidal thoughts in people suffering from anxiety disorders. These thoughts can be considered as a form of worry. Repetitive negative thoughts are conscious and sustained cognitive functions in which, the person is mostly focused on the negative dimensions of himself and the world around him, therefor it becomes difficult for the affected person to mentally control these thoughts. Researches show that these thoughts play an important role in causing psychological and physiological problems (3-8). In short, from a meta-cognitive perspective, mental disorder is caused by a certain toxic thinking style, called the cognitive-attentional syndrome. This syndrome has consequences that leads to the continuation of the negative emotions. The present study, investigated the relationship between the Cognitive-Attention Syndrome and the occupational stress levels of workers exposed to occupational accidents in a steel company.

\section{Methodology}

The present study was a descriptive correlational study. The statistical population consisted of employees of a steel company in Isfahan who were working in
2019. The total number of employees exposed to the accident was 600 people, and a sample of 300 people was randomly selected. Cognitive-attention syndrome and job stress questionnaires were used to collect data.

The Cognitive-Attention Syndrome Questionnaire contains 16 items, designed to assess the activation of Cognitive-Attention Syndrome (CAS). The first two questions, are designed to measure the anxiety level of the subject's and their attention level to the threatening cases, respectively. The next six items are related to the methods and strategies people use to deal with their negative emotions and thoughts. Responses to these eight items, are measured on an eight-point Likert scale from zero to eight. The next eight items rank the degree of belief, in each of the metacognitive beliefs about Cognitive-Attention Syndrome, on a scale of zero to 100 .

The job stress questionnaire was designed with 36 questions and a three-point response scale (completely correct to incorrect), to measure the job's stress level. The questionnaire is translated in Iran and assesses the matters of the workplace stress and pressure levels on an individual in relation to their work's nature, co-workers and supervisors. The validity of this questionnaire was reported to be acceptable and the reliability of the two halving and retesting methods were 0.69 and $0.72(\mathrm{p}<0.01)$, respectively.

\section{Result}

According to the study's findings, there was an impressive and significant correlation between the Cognitive-attentional syndrome and the job stress level $(r=0.94, p<0.01)$. The observed $F$ was also significant $(\mathrm{p}<0.0001)$, indicating a significant prediction and $88 \%$ of the variance related to the Cognitive-Attention Syndrome explained by the occupational stress. Regression coefficients indicate that the occupational stress $(\mathrm{p}=0.01, \mathrm{t}=42.49)$ can significantly predict and explain the variance of the cognitive-attentional syndrome.

Table (1) presents descriptive indicators (mean and standard deviation) related to the variables of the cognitive-attention syndrome and the job stress related to the research subjects.

As can be seen in Table (1), the mean (and standard deviation) of the research subjects suffering from the cognitive-attention syndrome was 80.46 (21.65) and the mean (and standard deviation) in the job stress was 74.64 (21.14).

In the following, we will deal with the findings of

Table 1. Descriptive indicators of research variables

\begin{tabular}{ccccc}
\hline Variable & The minimum & Maximum & Mean & standard deviation \\
\hline Cognitive-attention syndrome & 24 & 121 & $80 / 46$ & $21 / 65$ \\
Occupational Stress & 19 & 108 & $74 / 64$ & $21 / 14$
\end{tabular}


The Relationship between Cognitive-Attention Syndrome ...

Table 2. Correlation matrix between research variables

\begin{tabular}{ccc}
\hline Variable & Cognitive-attention syndrome & Job stress \\
\hline Cognitive-attention syndrome & 1 & $* * 0 / 94$ \\
Job stress & $* * 0 / 94$ & 1 \\
\hline$* * \mathrm{P}<0 / 01 \quad * \mathrm{P}<0 / 05$ & &
\end{tabular}

Table 3. Summary of the regression model and the analysis of the variance of job stress regression on cognitive-attention syndrome

\begin{tabular}{|c|c|c|c|c|c|c|c|c|c|}
\hline $\begin{array}{l}\text { Source of } \\
\text { variance }\end{array}$ & Total squares & $\begin{array}{l}\text { Degrees of } \\
\text { freedom }\end{array}$ & $\begin{array}{l}\text { Average } \\
\text { squares }\end{array}$ & $\mathrm{F}$ & $\begin{array}{c}\text { Significance } \\
\text { level }\end{array}$ & $\mathrm{R}$ & $\mathrm{R}^{2}$ & $\begin{array}{c}\mathrm{R}^{2} \\
\text { Moderator }\end{array}$ & S.E \\
\hline Regression & $96845 / 45$ & 1 & & & & & & & \\
\hline left over & $12442 / 77$ & 232 & $\begin{array}{c}96845 / 45 \\
53 / 63\end{array}$ & $1805 / 71$ & 0/0001 & $0 / 941$ & $0 / 886$ & $0 / 886$ & $7 / 32$ \\
\hline Total & $109288 / 2$ & 233 & & & & & & & \\
\hline
\end{tabular}

Table 4. Predictive coefficients of cognitive-attention syndrome based on job stress

\begin{tabular}{cccccc}
\hline Variable & B & SEB & Beta & T & Significance level \\
\hline Job stress & $0 / 964$ & $0 / 023$ & $0 / 941$ & $42 / 49$ & $0 / 0001$ \\
\hline
\end{tabular}

the data analysis in response to the research's theory.

To answer the hypothesis that there is a relationship between the cognitive attention syndrome and the job stress of employees exposed to accidents, Pearson correlation coefficient was used. The results are presented in Table (2).

As can be seen in table (1), there is an impressive and significant correlation between the cognitiveattention syndrome and the job stress $(r=0.094$, $\mathrm{p}<0.01$ ). Multivariate regression analysis was also used. To determine the effect of the job stress on the variance of the cognitive-attention syndrome, the job stress as a predictor of the variables and the cognitive-attention syndrome as a criterion variable, were included in the regression equation. The analysis' results of the variance and statistical characteristics of the regression are described below.

Based on the results of the above tables, the observed $\mathrm{F}$ is significant ( $\mathrm{p}<0.0001)$, which indicates that the prediction is significant and $88 \%$ of the variances related to the cognitive-attention syndrome are explained by job stress. Regression coefficients show that the variable of the job stress $(p<0.01, t=$ 42.49) can predict and explain the variance of the cognitive-attention syndrome.

\section{Conclusions}

As mentioned in the research's findings section, it can be concluded that there is a significant relationship between the two research variables and the job stress has the ability to predict the cognitiveattention syndrome in employees that are exposed to occupational accidents. These findings are consistent with other studies related to the relationship between the cognitive-attention syndrome and the emotional disorders. Explaining this result, it can be said that the inhibition mechanism seems to, lead to the screening of the information conducted from the normal people and focuses on the target stimuli or related information in people with the job stress, leading to defects. It is inhibited and does not perform the filtering task well. It can be said that, in employees with job stress, anxiety and stress is an undesirable emotional experience, that indicates the existence of various threats or lacks, and when people respond to them by changing the pattern of attention and thinking, they become stable and problematic. These people are not able to recognize that their attention is locked on themselves, their thoughts and their feelings. This process increases and perpetuates negative feelings and beliefs about themselves. Unfortunately, people are usually unaware of this process and find it difficult to change. When an event stops this process, if they pay attention to its consequences, they will notice the negative and inefficient effects of extreme engagement with their symptoms and thoughts. Therefore, it can be concluded that there is a significant relationship between the two variables of the study, and the job stress has the ability to predict the cognitive-attention syndrome in the employees 
exposed to occupational accidents. Researchers also faced problems and limitations in conducting this study, such as the limited sample of research from a company and the fact that the data collection was a questionnaire. Therefore, considering the importance of attention control and cognitive-attention flexibility in employees exposed to occupational accidents, the following suggestions are made. Repeating similar studies with larger sample sizes, it is suggested that structured interviews be used in the future researches. It is also suggested, that psychological therapies that emphasize mindfulness and trainings to reduce job stress in employees exposed to occupational accidents be taken into consideration.

\section{Conflict of Interest}

"The authors declare that there are no conflicts of interest regarding the publication of this manuscript".

How to cite this article:

Zohreh Hashemi, Taghi Shahjoee. The Relationship between Cognitive-Attention Syndrome and Occupational Stress of Workers Exposed to Occupational Accidents. Iran Occupational Health. 2021 (01 March);18:5. 


\section{رابطه سندرم شناختى - توجهى با استرس شغلى در كار كنان شاغل در معرض حوادث شفلى}

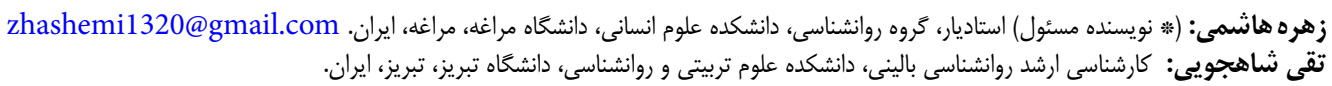

جكيده

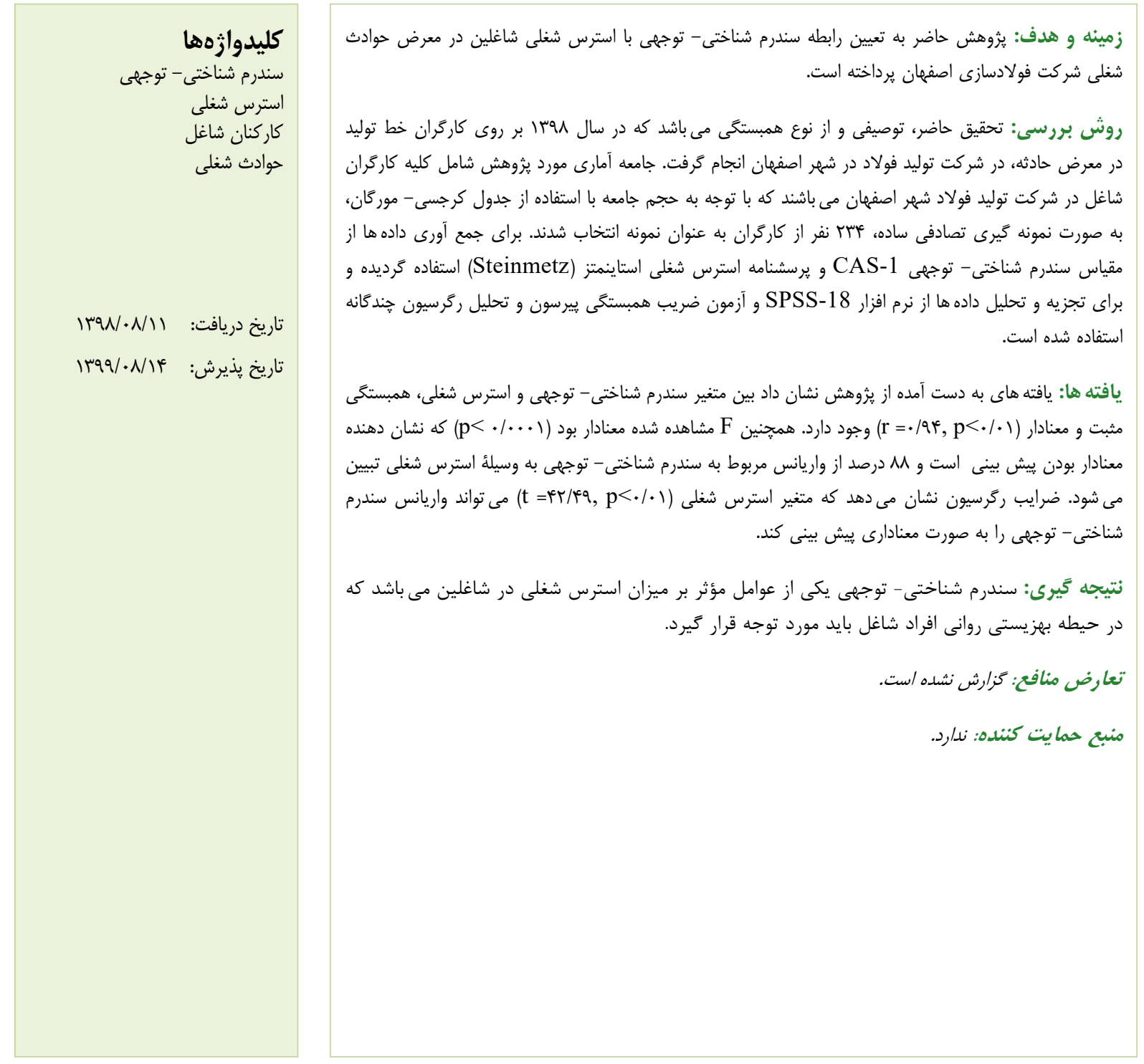

شيوه استناد به اين مقاله:

Zohreh Hashemi, Taghi Shahjoee. The Relationship between Cognitive-Attention Syndrome and Occupational Stress of Workers Exposed to Occupational Accidents. Iran Occupational Health. 2021 (01 March);18:5. 
مفهومى، سندرم شناختى - توجهى شامل توجه سو ئيرانه

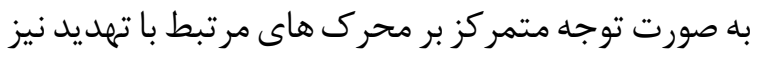

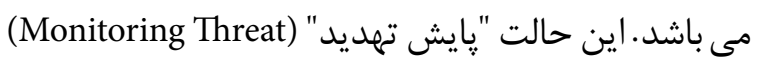

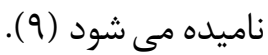

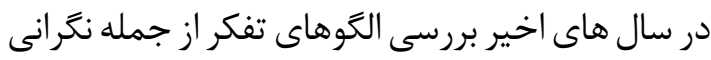

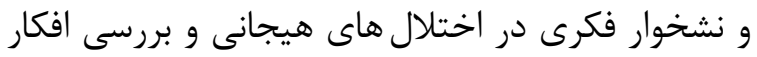

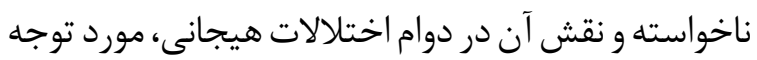

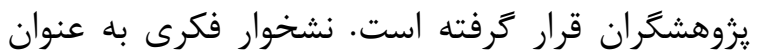

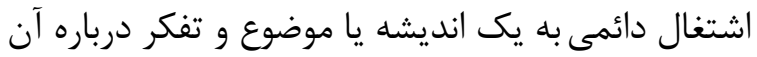

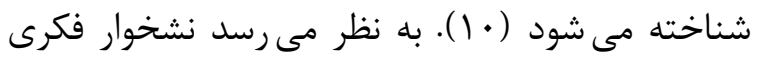

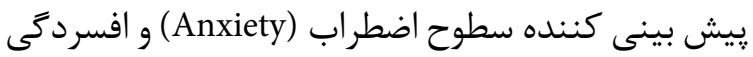

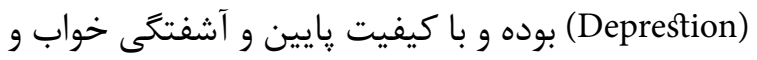

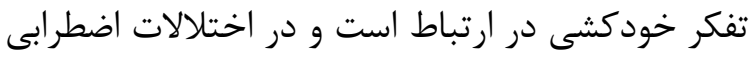

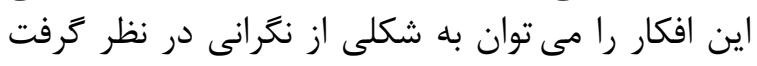

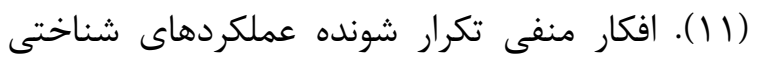

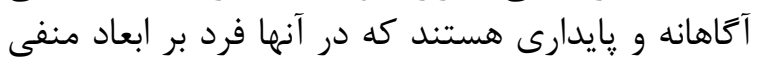

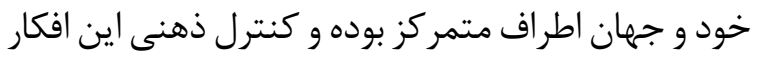

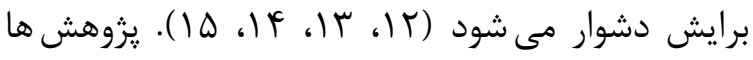

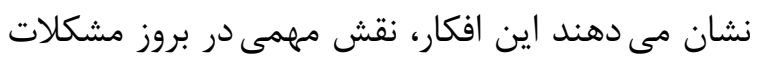

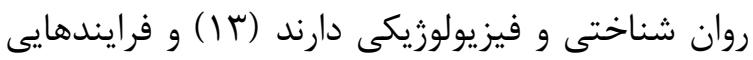

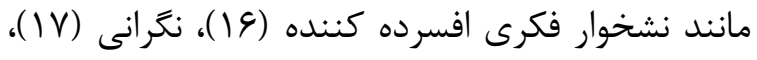

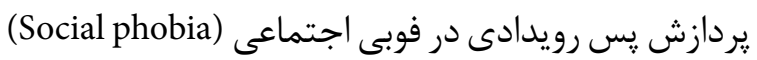

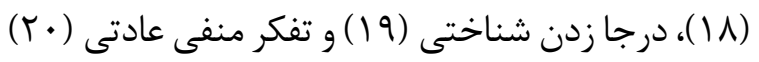

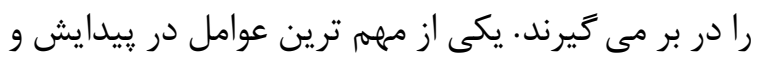

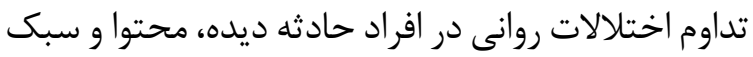

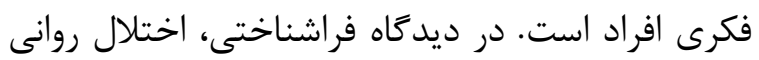

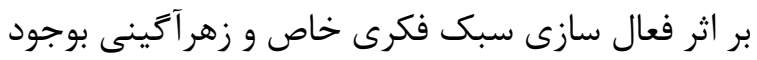

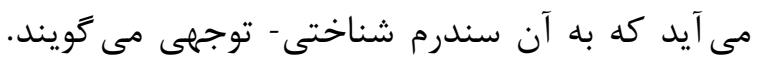

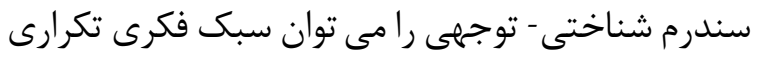

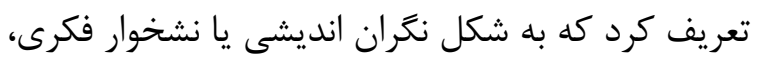

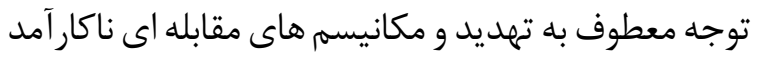

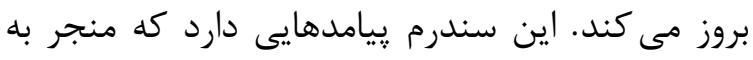

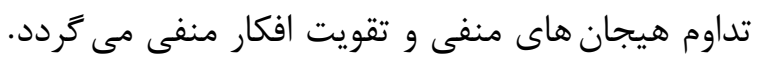

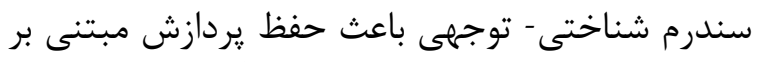

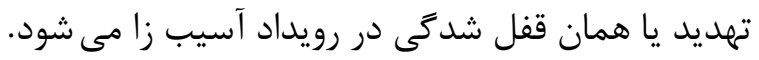

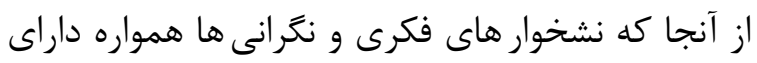

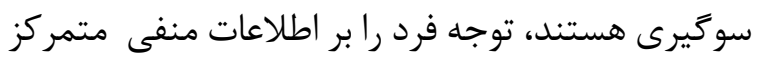

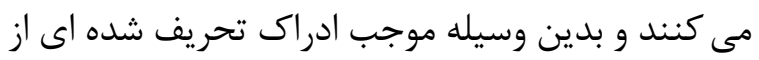

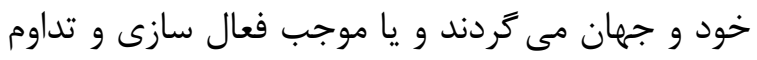

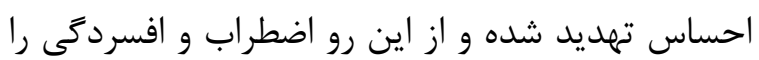

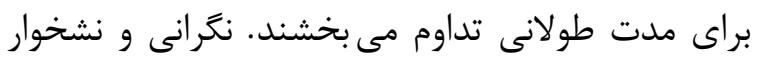

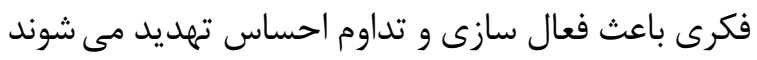

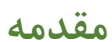

افراد شاغل در محيط هاى صنعتى به دليل ماهيت

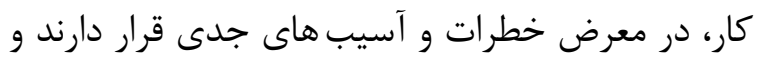

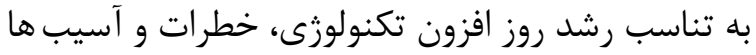

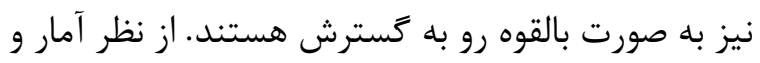

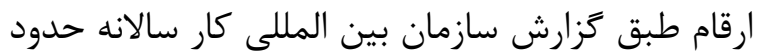

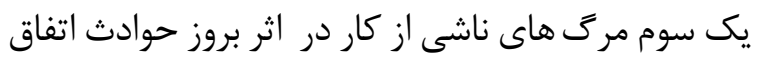

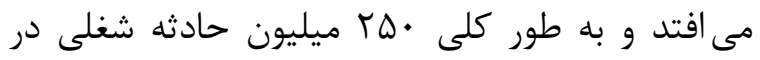

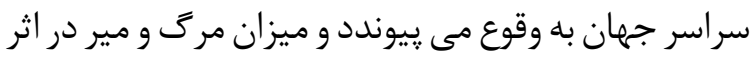

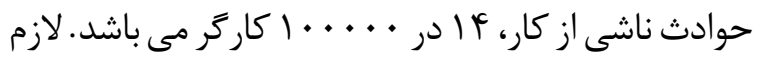

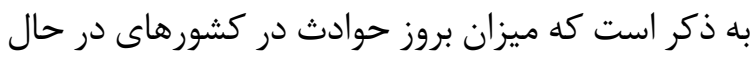

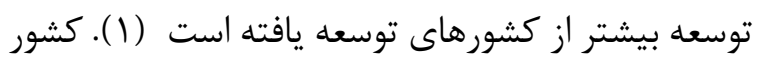

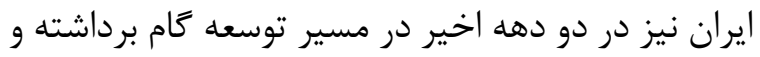

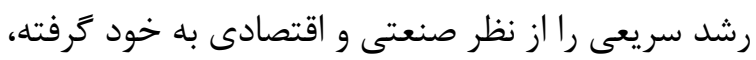

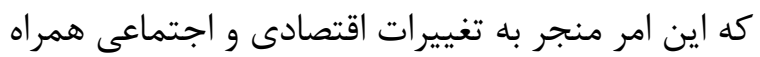

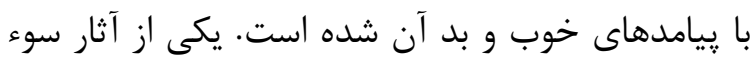

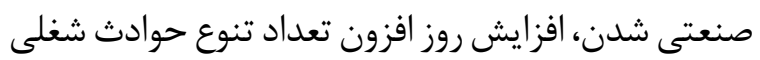

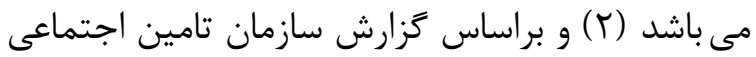

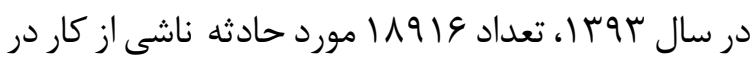

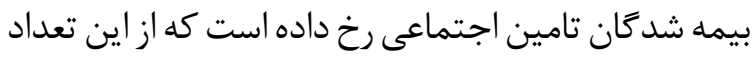

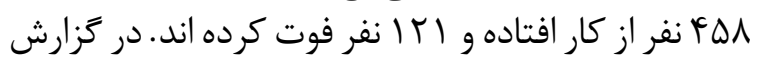

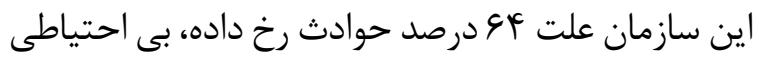

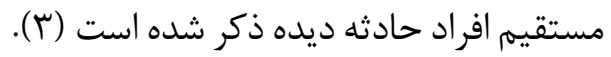

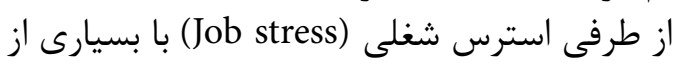

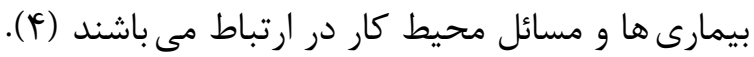

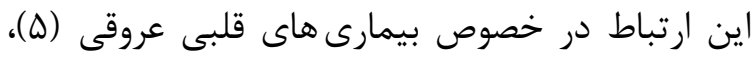

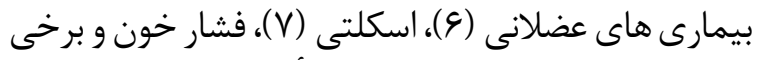

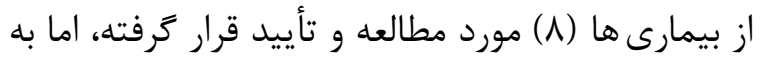

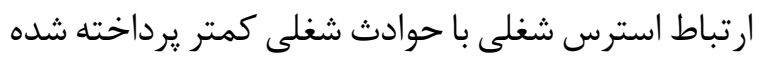

در رويكرد فراشناختى (Metacognitive) به سبك تأن

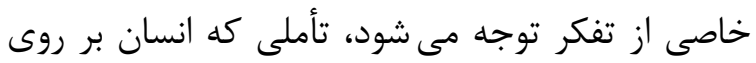

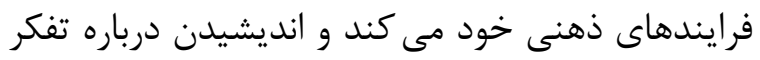

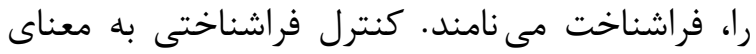

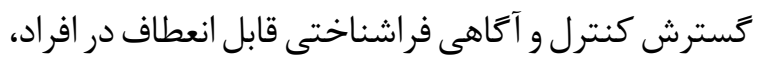

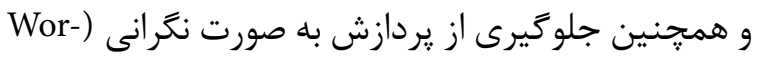

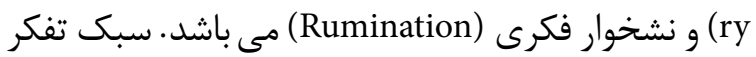

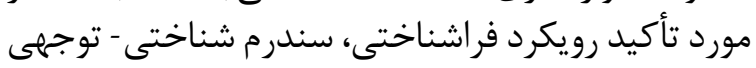
(Cognitive Attention Syndrome-CAS)

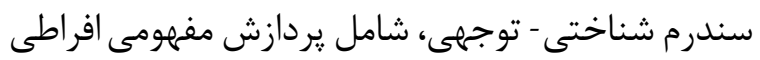
به صورت نكرانى، نشخوار فكرى، توجنه

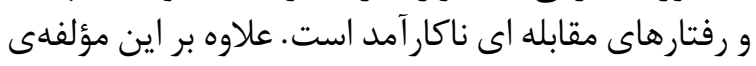




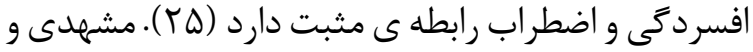

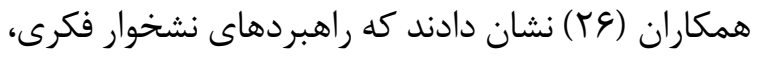

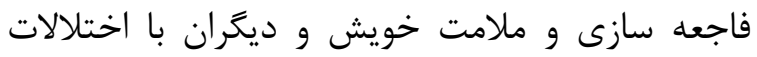

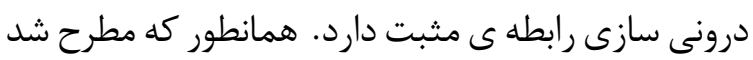

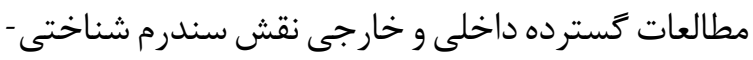

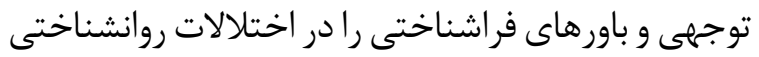

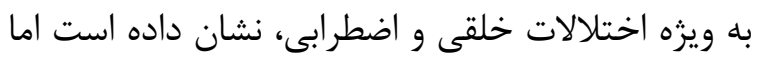

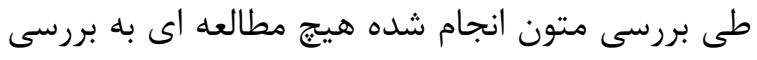

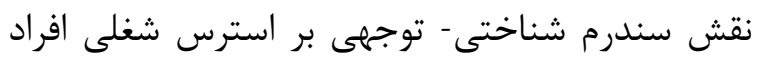

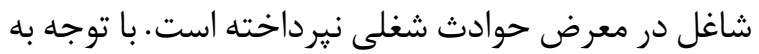

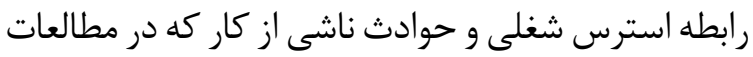

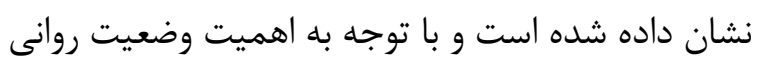

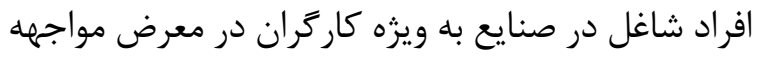

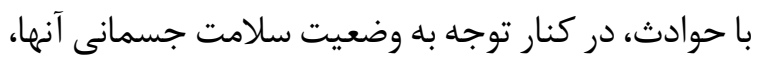

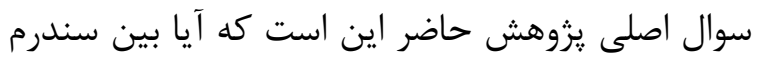

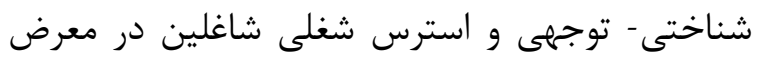
حوادث، رابطه ایى وجود دارد؟ توني

\section{روش بررسى} مطالعه حاضر از نظر هدف كاربردى و از آنجايى كه

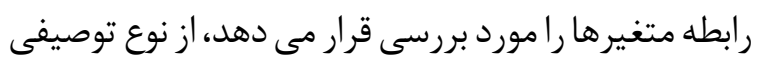

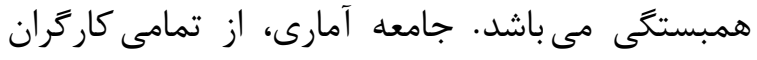

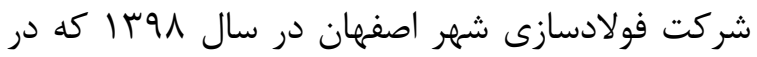

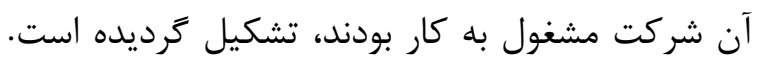

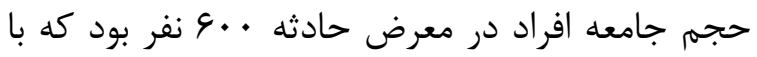

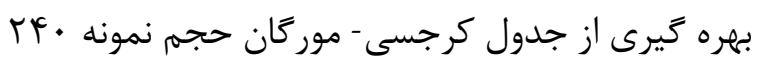

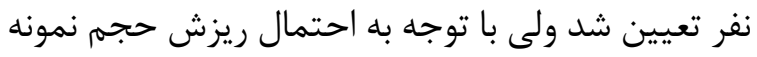

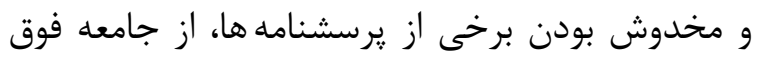

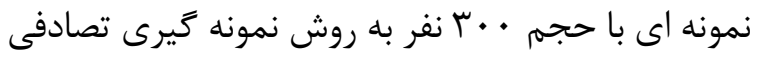

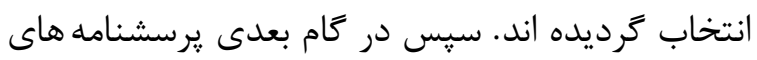

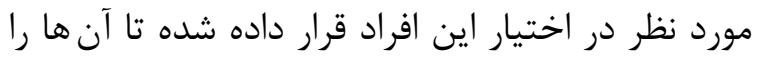

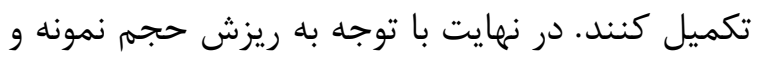

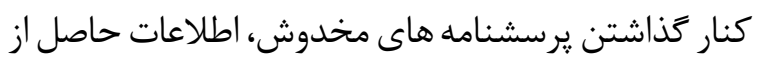

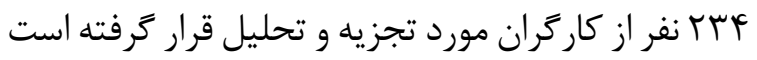

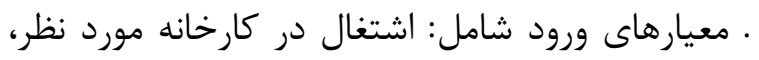

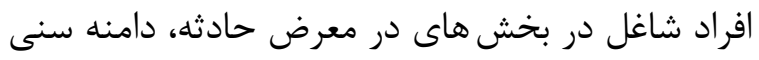

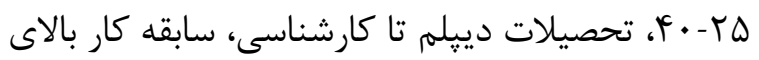

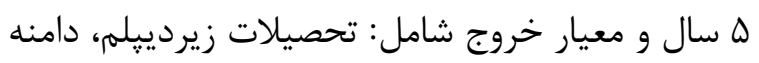

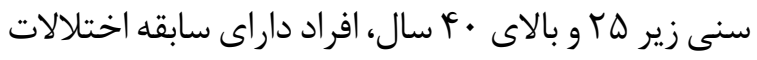

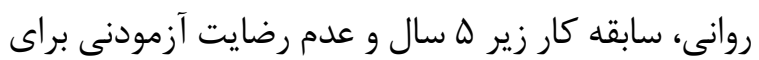

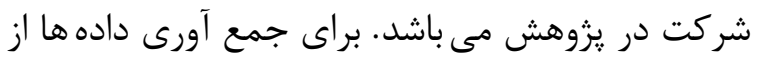

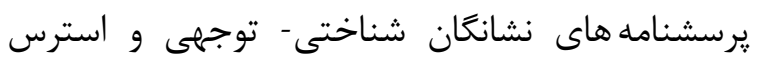

و اين كار اضطراب و افسردگى را به حالت هاى ماند

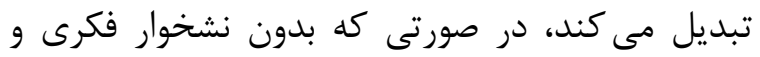

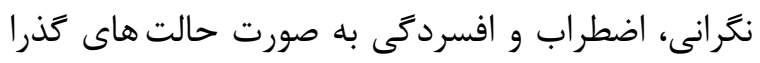

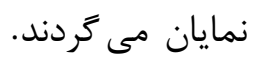

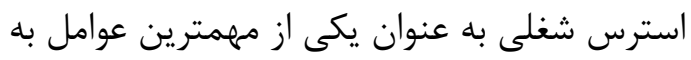

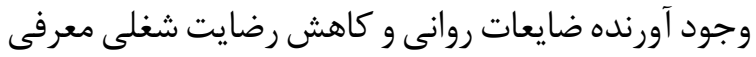

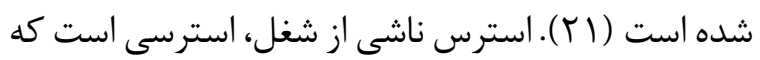

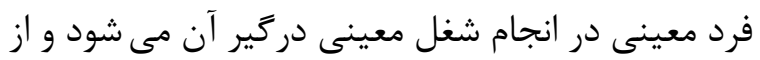

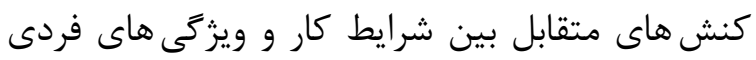

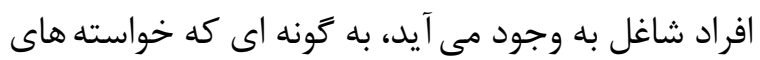

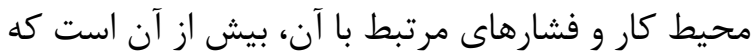

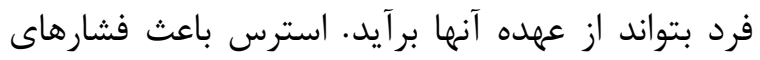

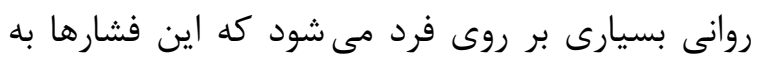

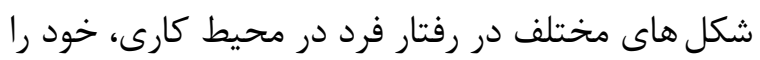

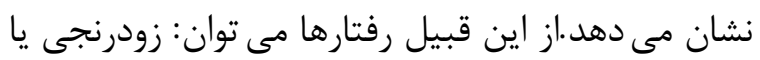

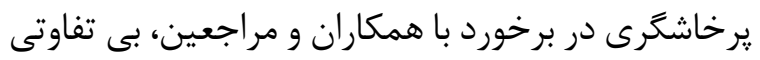

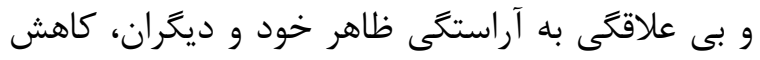

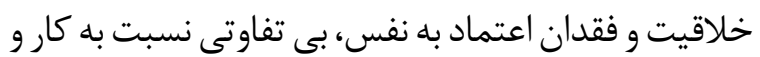

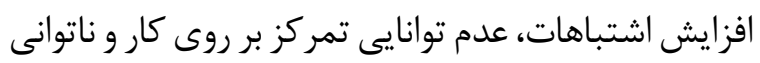

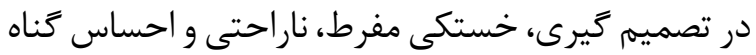

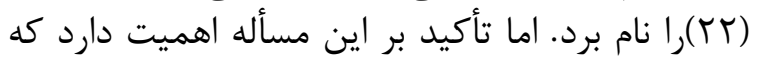

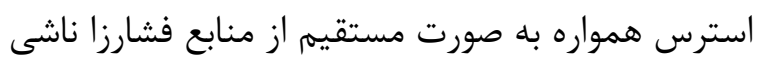

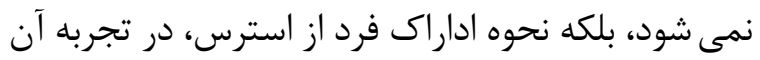

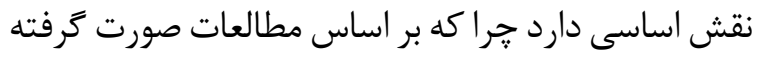

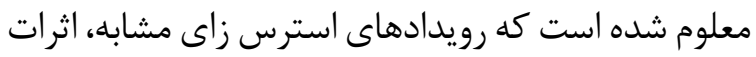

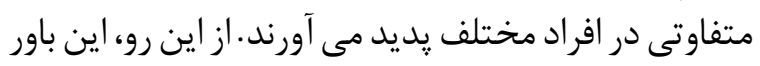

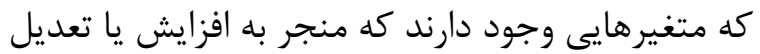

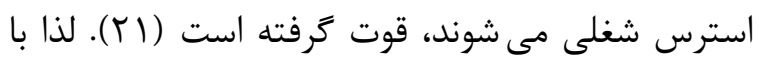
توجه به اين مطالب، استرس شغلى شئى كاركنان از مسائلى

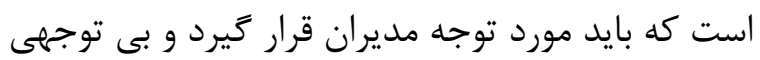

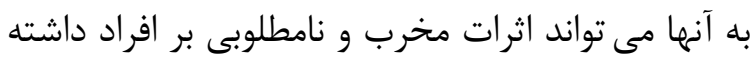

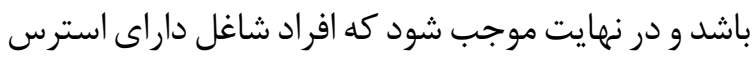

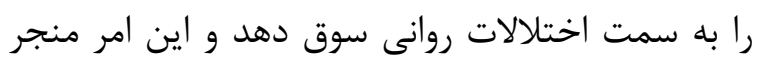

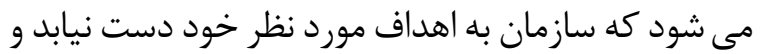

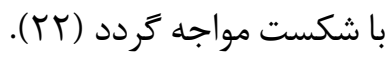

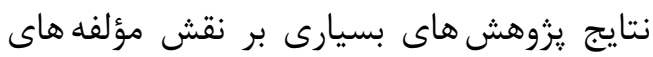

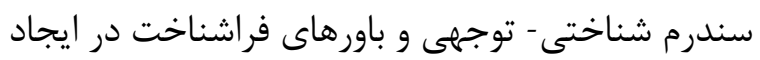

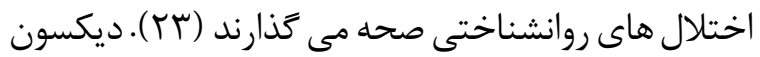

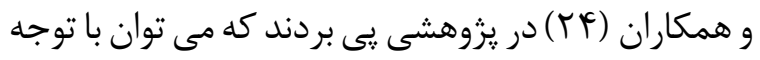

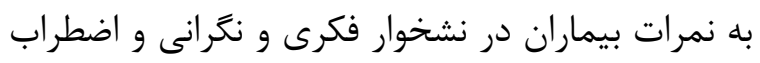

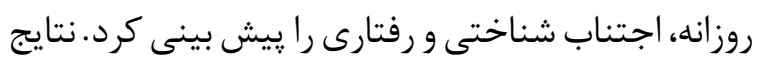

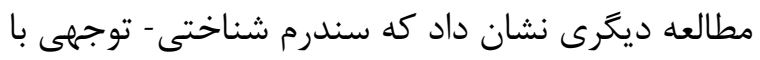


ماهيت كار، همكاران و سريرستان مى يردازد. ماده هاي

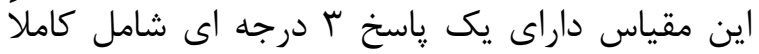

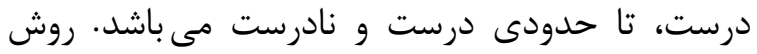

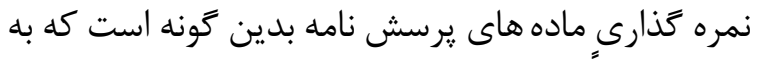

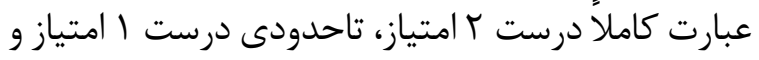

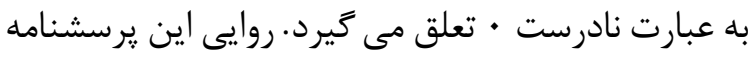

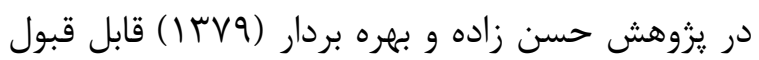

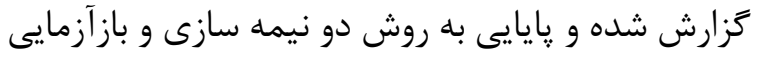

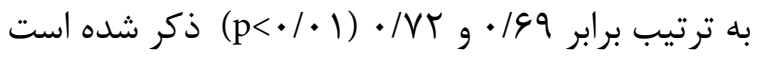

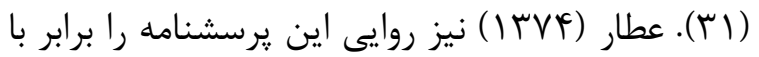

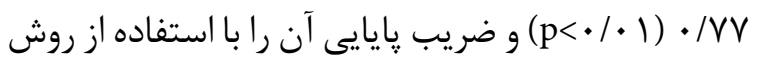

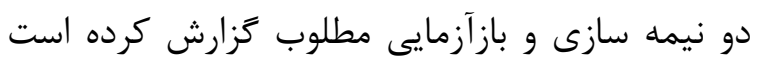

\section{يافته ها}

در جدول البه اطلاعات جمعيت شناختى نمونه هاى

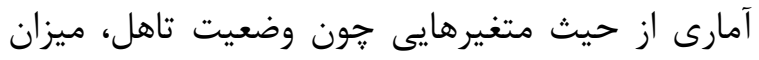

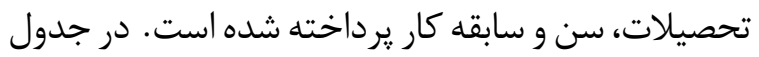

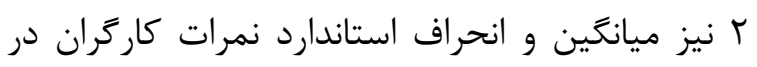
معرض خطر در متغيرهاى سندرم شناختى - توجهى و و استرس شغلى ارائه گرديده است.

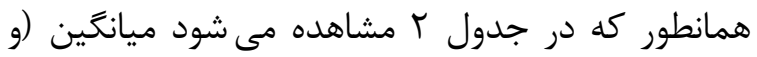

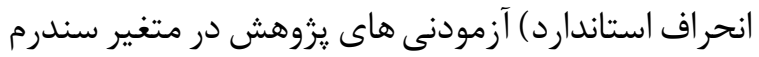

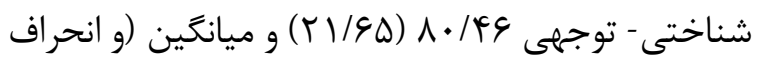

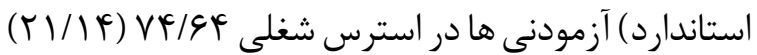

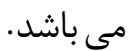

شغلى استاينمتز استفاده شده است. به منظور گردآورى

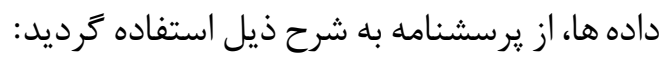

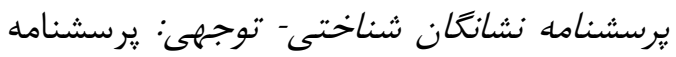

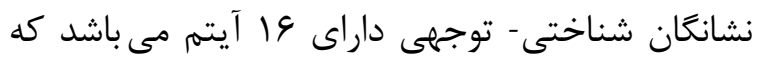

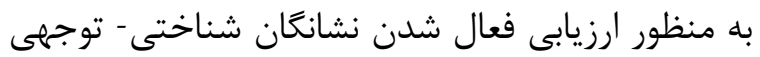

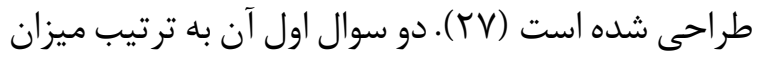

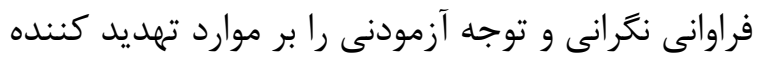

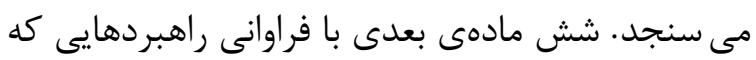

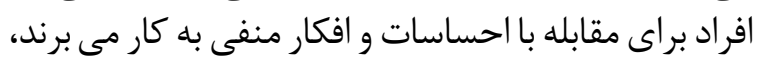

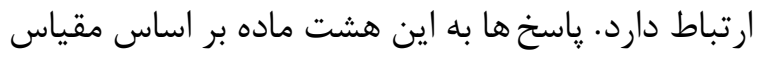

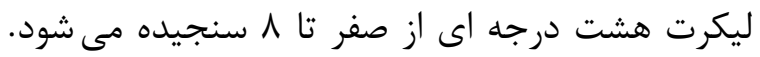

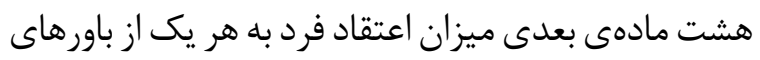

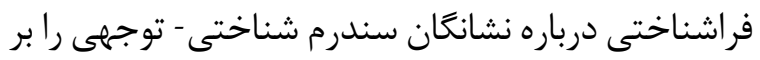

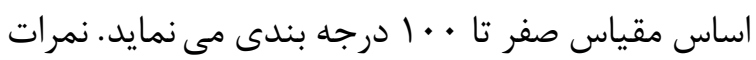

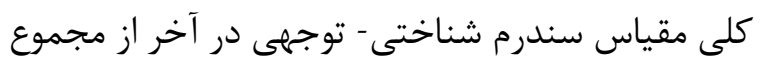

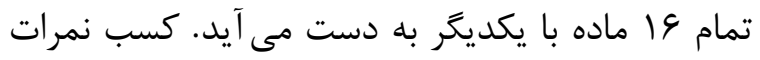

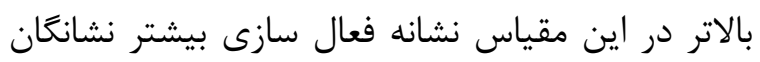

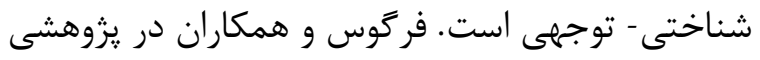

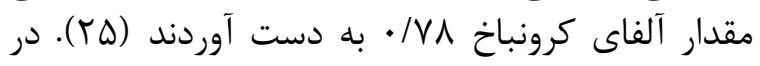

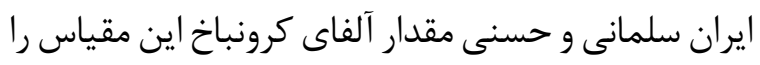

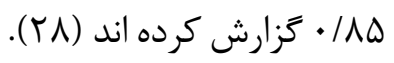

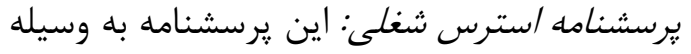

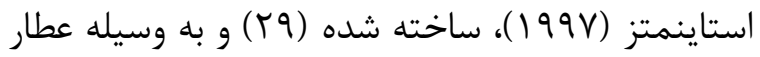
(I IVYF)

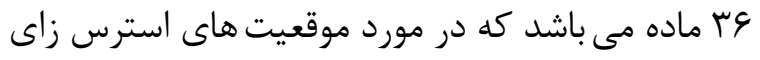

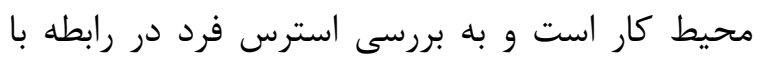

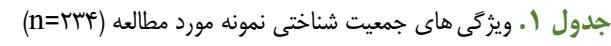

\begin{tabular}{|c|c|c|c|}
\hline درصد & فراوانى & شاخص & متغير \\
\hline$\Delta r / \Gamma^{\leftarrow}$ & ITS & متاهل & \multirow{3}{*}{ وضعيت تاهل } \\
\hline $\mid q / 8$ & 1.9 & مجرد & \\
\hline $1 . . \%$ & Tre & كل & \\
\hline$M E / T$ & $\wedge$. & دييلم & \multirow{4}{*}{ ميزان تحصيلات } \\
\hline$r V / \varphi$ & $M$ & كاردانى & \\
\hline TN/T & 99 & كارشناسى & \\
\hline $1 . .1$ & Tre & كل & \\
\hline$r q / \Delta$ & 99 & ه تا • r سال & \multirow{4}{*}{ سن } \\
\hline$F T / r$ & 99 & וץ تا هץ سال & \\
\hline TN/T & 99 & عب تا •r سال & \\
\hline $1 . . \%$ & THE & كل & \\
\hline$r \cdot / r$ & 94 & ه سال & \multirow{4}{*}{ سابقه كار } \\
\hline щ & $M$ & ع تا •ا سال & \\
\hline$r M / \Lambda$ & $\Delta F$ & 1ا تا ها سال & \\
\hline $1 . . \%$ & rme & كل & \\
\hline
\end{tabular}


رابطه سندرم شناختى -توجهى با استرس شغلى ...

جدول ץ. شاخص هاى توصيفى ميانكين و انحر اف استاندارد متغيرهاى مورد يخوهش

\begin{tabular}{|c|c|c|c|c|}
\hline انحراف استاندارد & ميانكين & بيشينه & كمينه & متغير \\
\hline$r I / \varepsilon_{0}$ & 1.149 & $|r|$ & tre & سندرم شناختى - توجهى \\
\hline$r M / T F$ & $V v^{c} / q^{c}$ & 1.1 & 19 & استرس شغلى \\
\hline
\end{tabular}

كاركران در معرض خطر را بِيش بينى مى كند. مقدار بتا

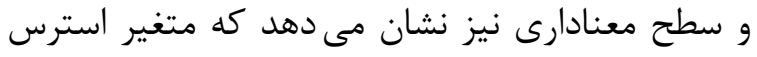

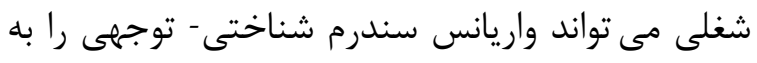
صورت معنادار بيش بينى و تبيين نمايد.

هدف يزوهش حاضر بررسى ارتباط بين سندرم

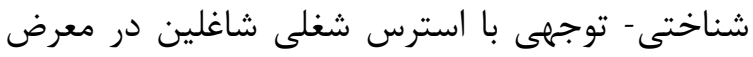

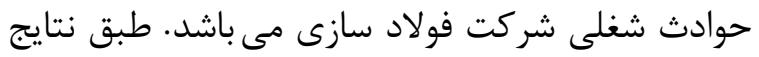

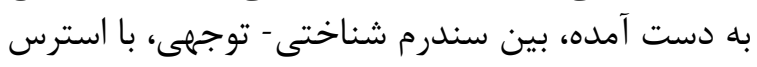

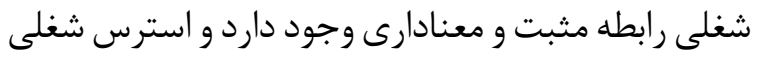

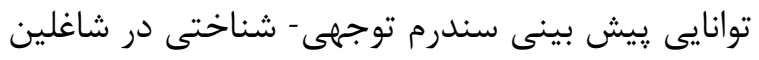

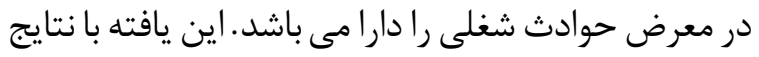

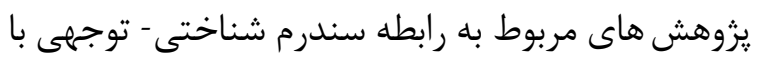

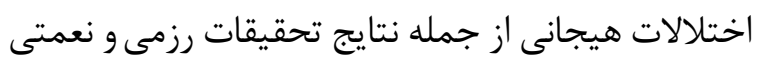

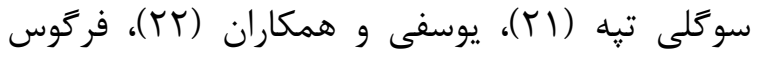

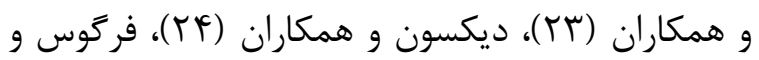

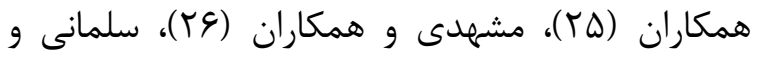

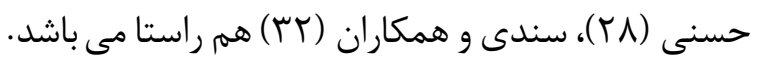

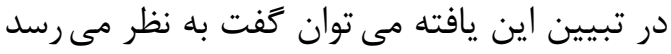

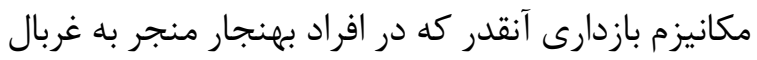

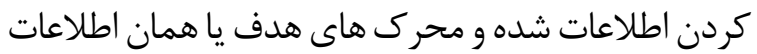
مربوط را در كانون توجه فرد قرار مى دهد، در افر افراد داراى اطلات

\begin{tabular}{|c|c|c|}
\hline استرس شغلى & سندرم شناختى - توجهى & متغير \\
\hline.$/ 9 \Upsilon^{*}$ 䄅䄅 & 1 & سندرم شناختى - توجهى \\
\hline 1 & $\cdot / 94 * *$ & استرس شغلى \\
\hline
\end{tabular}

به منظور نشان دادن ارتباط بين متغيرهاى سندرم

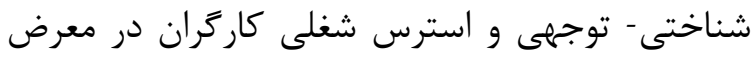

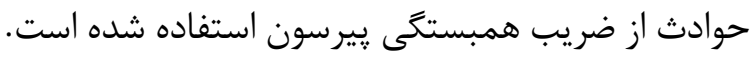

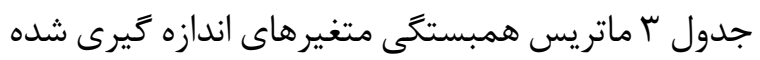
را نشان مى دهد.

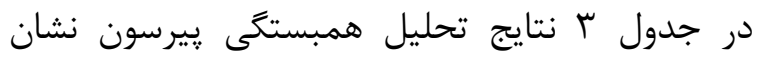

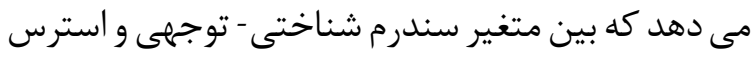

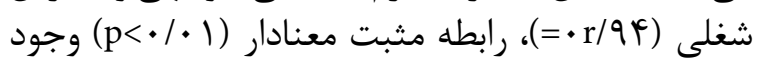
دارد. - ماري

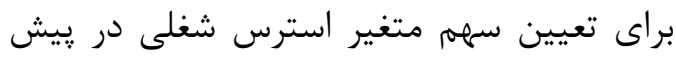

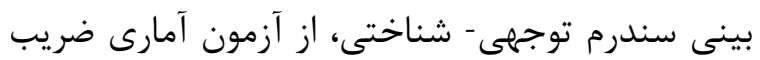

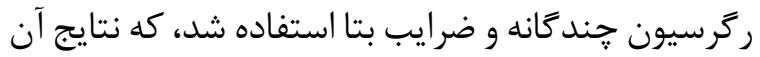

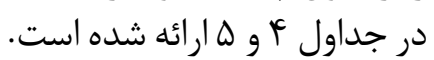

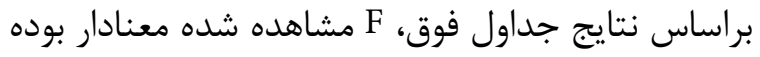

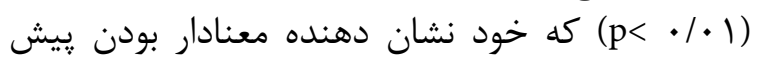

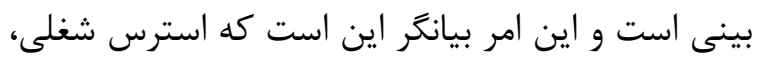

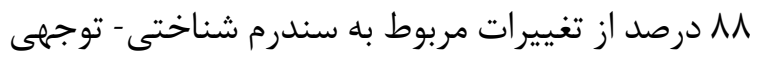

جدول ع. شاخص هاى آمارى و نتايج تحليل ركرسيون مريوط به نقش استرس شغلى بر سندرم شناختى - توجهى

\begin{tabular}{|c|c|c|c|c|c|c|c|c|c|}
\hline S.E & 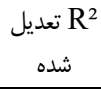 & $\mathrm{R}^{2}$ & $\mathrm{R}$ & معنادارى & $\mathrm{F}$ & مجذورها & آزادى & مجدمورعا & واريانس \\
\hline & & & & $\cdot|\cdots|$ & $M \cdot \Delta / V \mid$ & $q S \Lambda F \Delta / F \Delta$ & 1 & $q \varepsilon \wedge F \Delta / \digamma \Delta \Delta$ & ركرسيون \\
\hline \multirow[t]{3}{*}{$V / r r$} & $\cdot / M S$ & $\cdot \mid M N S$ & $. / 94 \mid$ & & & & & & \\
\hline & & & & & & $\Delta \Psi / \mathcal{G}$ & rTr & ITEAT/VV & باقيمانده \\
\hline & & & & & & & Trז & $1.9 r M / r$ & مجموع \\
\hline
\end{tabular}

جدول ه. شاخص هاى آمارى و ضرايب مربوط به ييش بينى سندرم شناختى- توجهى از روى استرس شغلى

\begin{tabular}{|c|c|c|c|c|c|}
\hline سطح معنادارى & $\mathrm{T}$ & Beta & SEB & ضريب B & متغير \\
\hline.$/ \cdots 1$ & $p r / 4 q$ &.$/ 941$ & Tr/. &.$/ 994$ & استرس شغلى \\
\hline
\end{tabular}


و باورهاى تهديدآميز بودن جنبه هاى مختلف زندگى در در

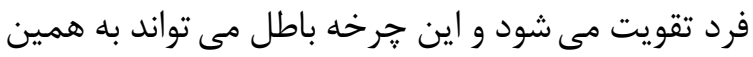

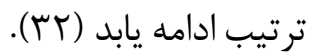

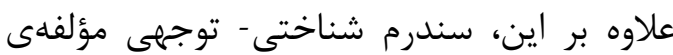

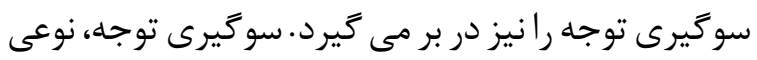

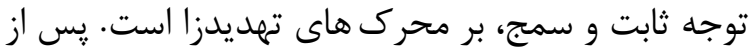

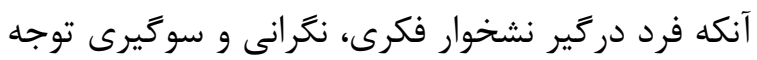

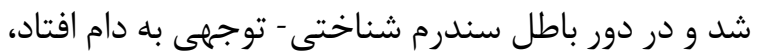

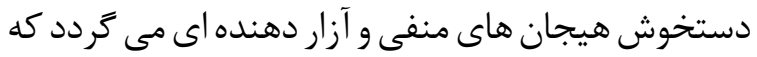

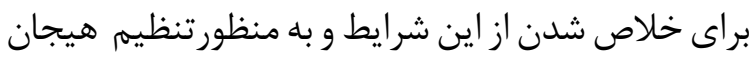

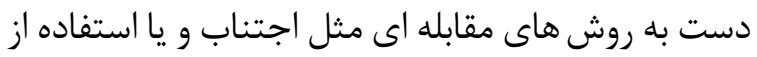

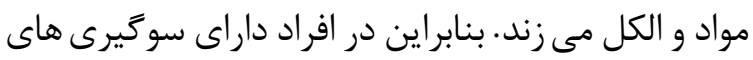

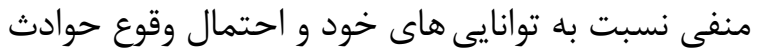

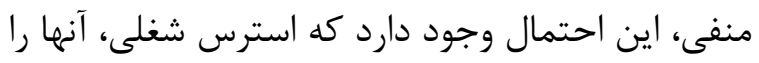

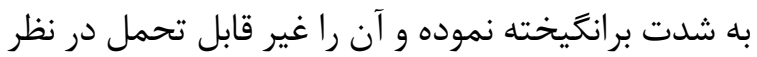

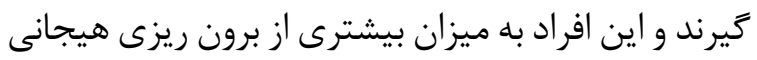

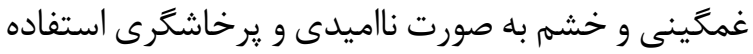

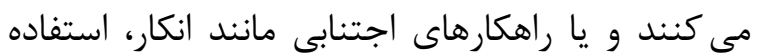

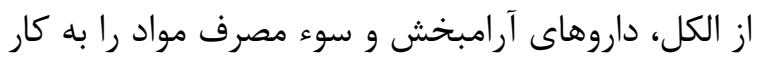

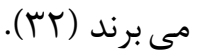

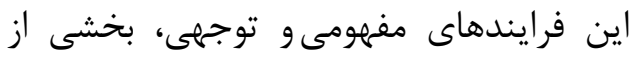

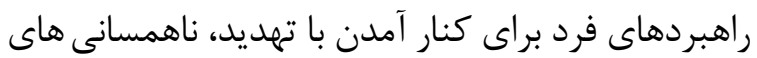

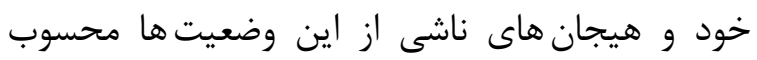

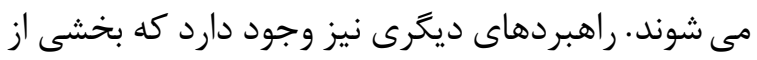

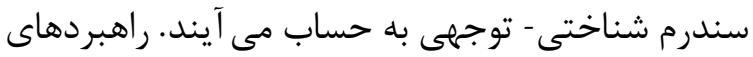

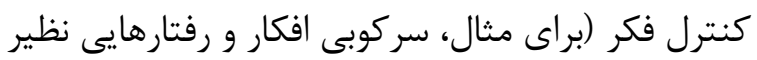

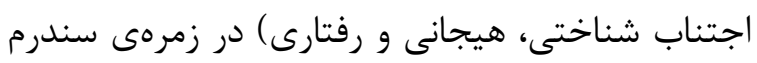

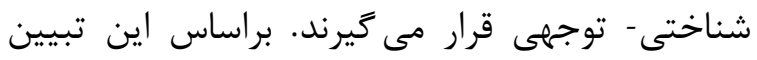

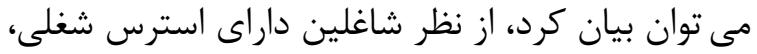

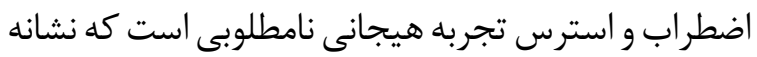

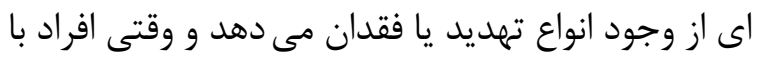

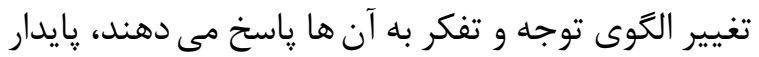

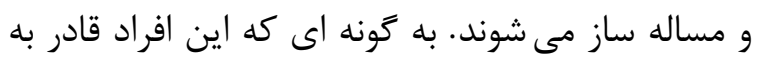

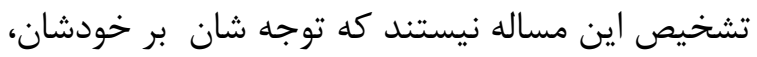
تفكرشان و احساس شان قفل شان شده است. اين فران فرايند

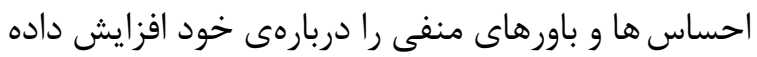

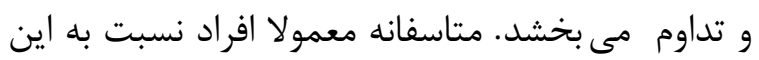

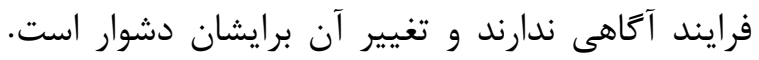

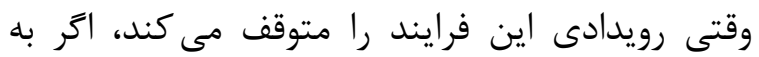

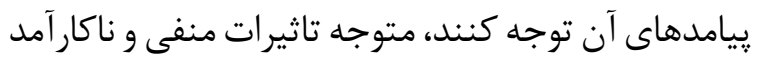

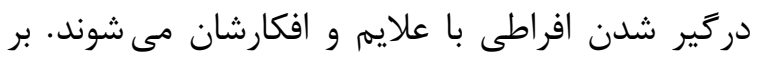

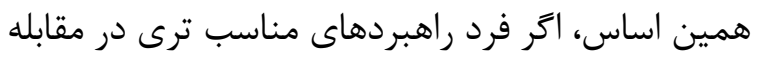

استرس شغلى، منجر به نقص بازدارى شده و جندان

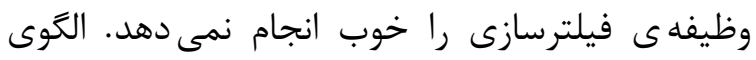

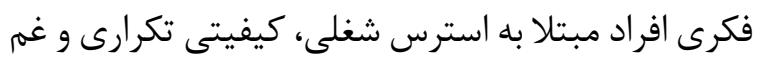

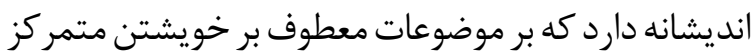

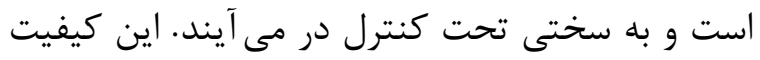

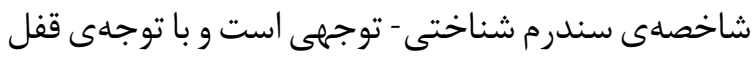

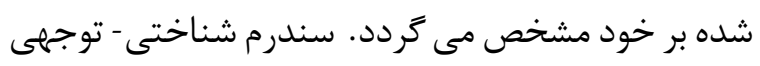

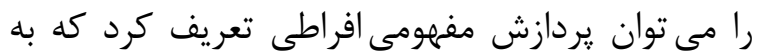

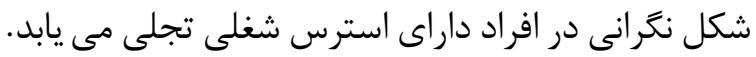

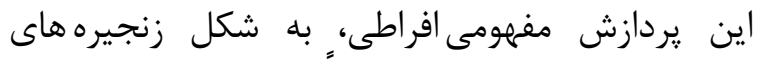

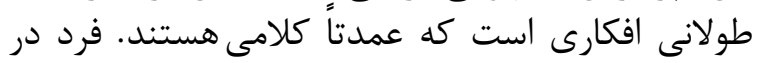

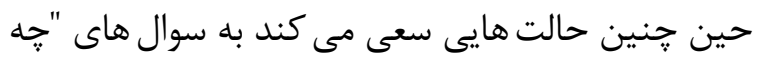

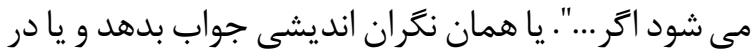

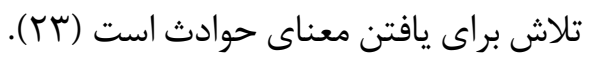

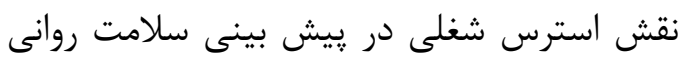

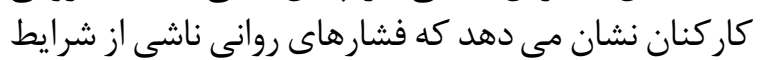

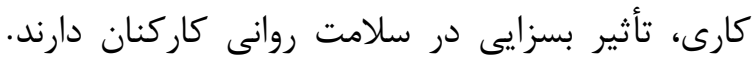

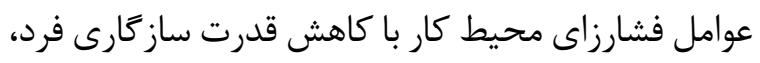

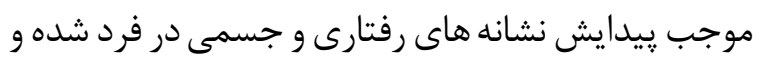

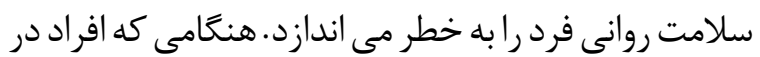

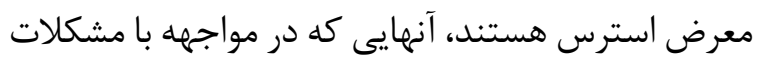

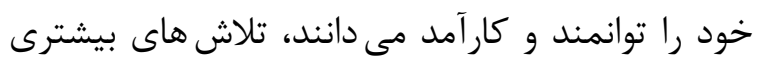

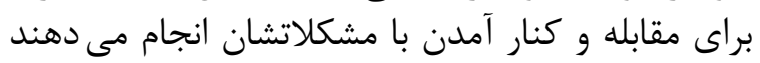

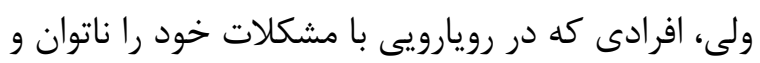

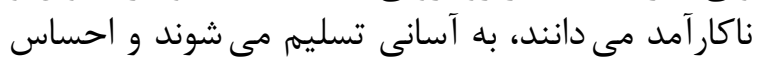

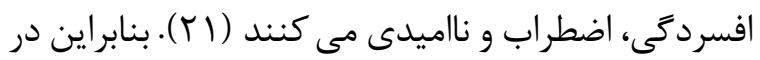

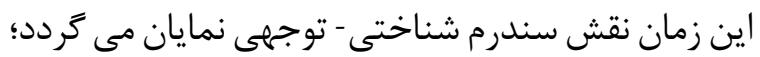

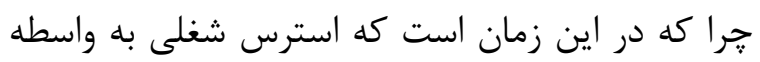

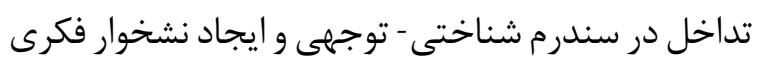

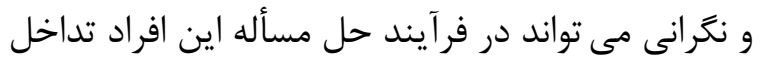

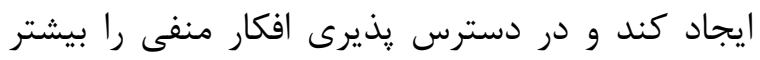

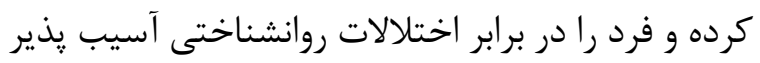

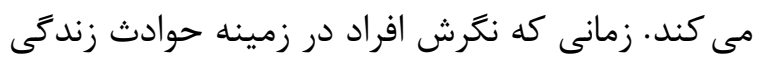

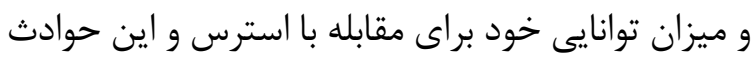

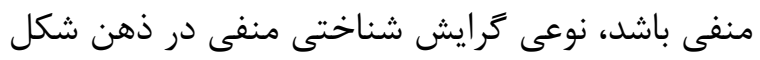

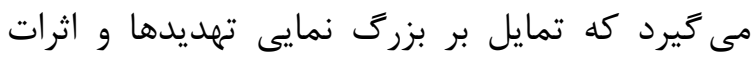

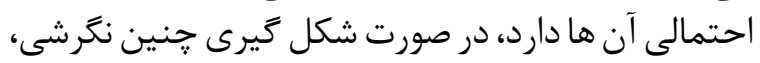

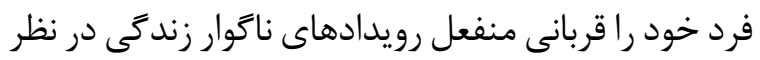

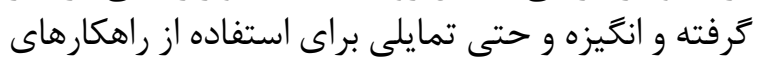

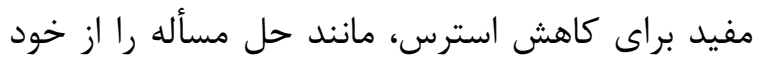

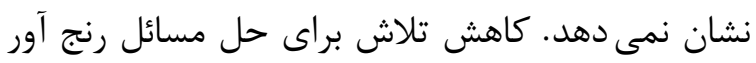

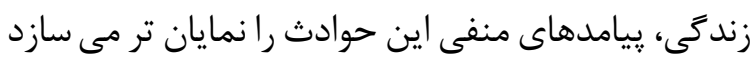


اين كه آيا همبستخى هاى بدست آمده در اين يزوهش در

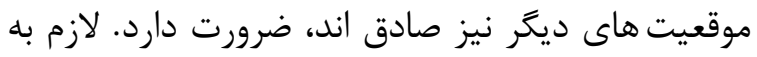

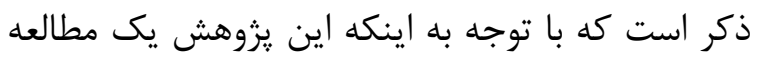

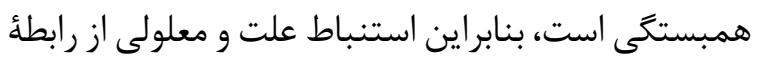

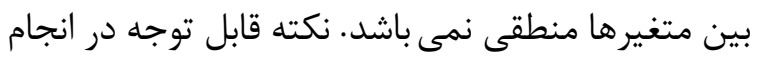

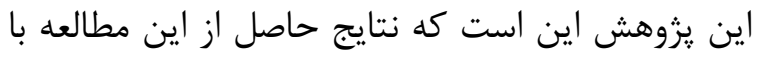

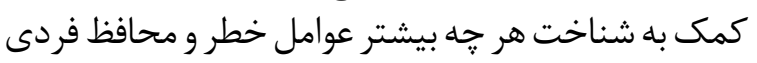

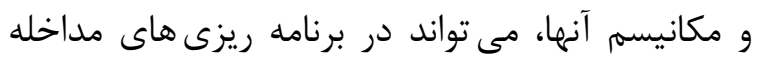

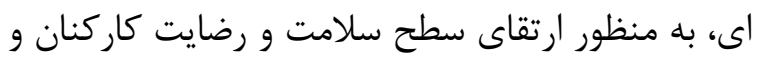

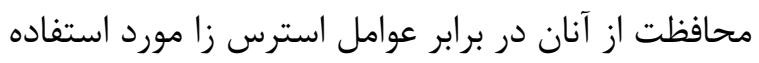

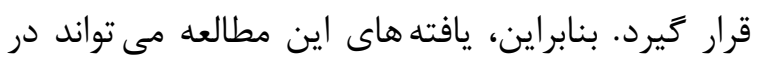

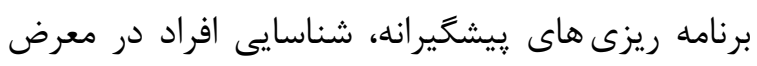

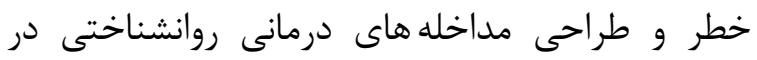
محيط هاى كارى مفيد باشد.

$$
\begin{aligned}
& \text { تقدير و تشكر } \\
& \text { از همكارى كليه مسئولان و كار كنان شركت فئل فئلادسازى }
\end{aligned}
$$

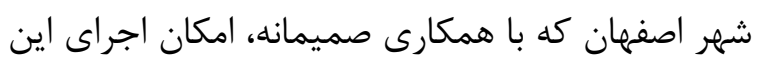

$$
\begin{aligned}
& \text { يزوهش را فراهم نمودند، سياسگزارى مي مى كنيم. }
\end{aligned}
$$

\section{References}

1. O'Toole M. The relationship between employees' perceptions of safety and organizational culture. J Safety Res. 2002;33(2):231-43.

2. Halvani G, Fallah H, Barkhordari A, Khoshk Daman R, Behjati M, Koohi F. A Survey of causes of occupational accidents at working place under protection of Yazd Social Security Organization in 2005. Iran Occupa Health J. 2010;7(3):22-9. [Persian]

3. Mortazavi SB, Asilian H, Ostakhan M. The relationship between safety climate factors and workers behavior working in potentially dangerous situations in height among construction workers. Iran Occupa Health J. 2011;8(1):51-60. [Persian]

4. Jeanne-Marie R, Stacciarini RN, Bartholomeu TT. Occupational stress and constructive thinking: health and job satisfaction. J Adv Nur. 2004; 46(5):480-7.

5. Greiner BA, Krause N, Ragland D, Fisher JM. Occupational stressors and hypertension: a multi-method study using observer-based job analysis and self-reports in urban transit operators. Soc Sci Med. 2004;59(5):1081-94.

6. Huang GD, Feuerstein M, Sauter SL. Occupational stress andwork-related upper extremity disorders:concepts and models.Am J Ind Med. 2002;41(5):298-314.

7. Stanfeld SA. The problem with stress, minds, hearts and disease. Int J Epidemol. 2002;(31):11:13-16.

8. Landsbergis PA, Schurman SJ, Israel BA, Schnall PL, Hugentobler MK, Cahill J, et al. Job stress and
با استرس و حوادث به كار برد از سلامت روانى بالاترى

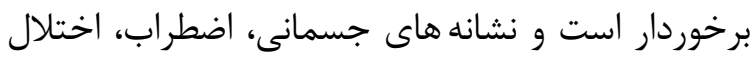

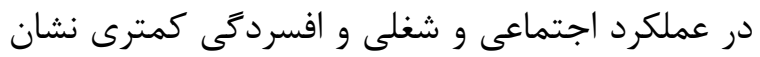

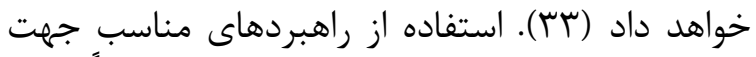

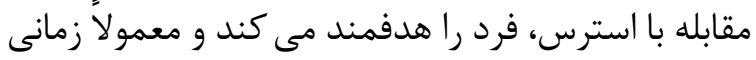

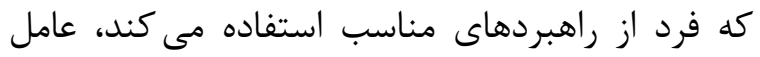

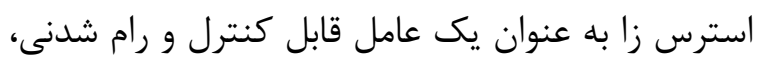

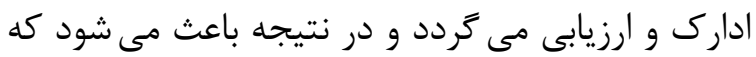

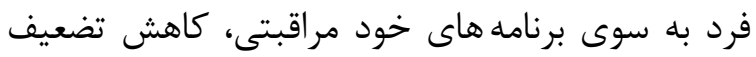

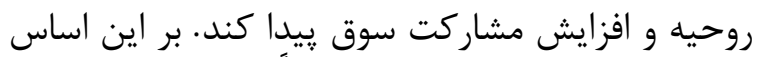

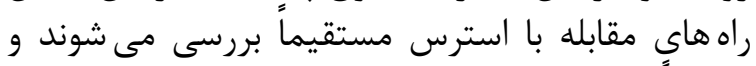

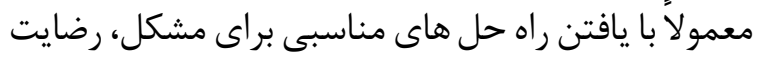

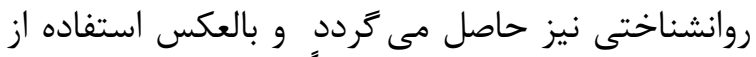

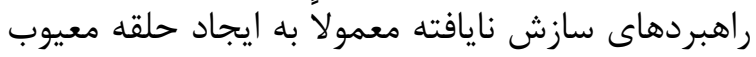

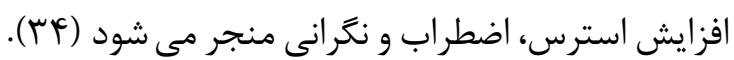

$$
\text { نتيجه كَيرى }
$$

به طور كلى مى توان اظهار كرد كه سندرم شناختى - تونه

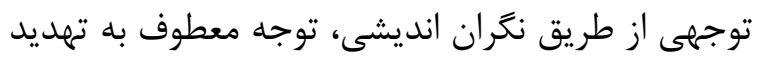

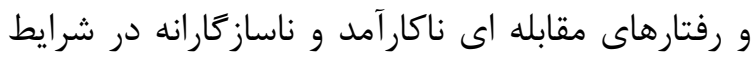

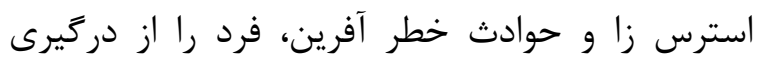

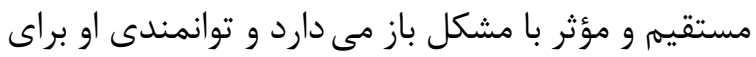

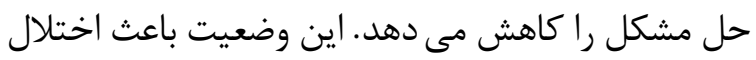

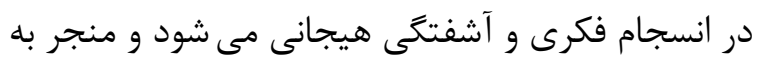

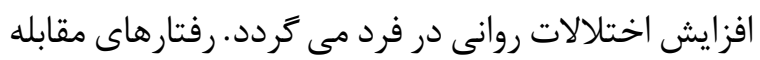

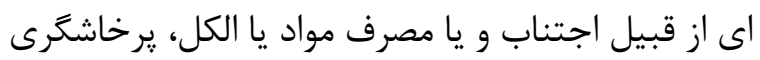

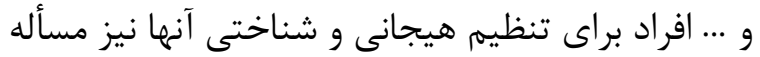

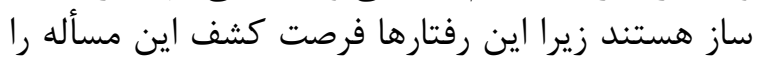

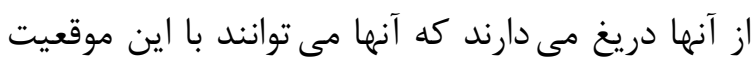

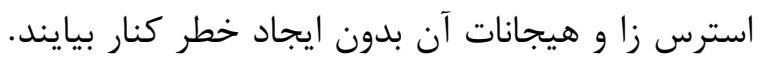

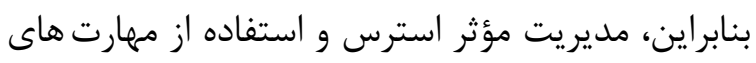

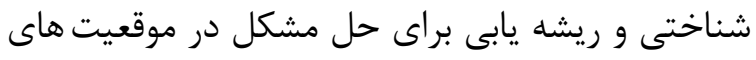

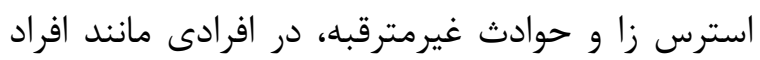

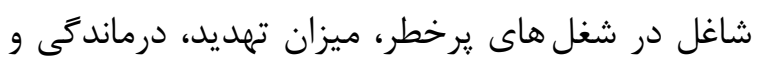

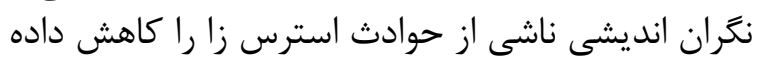

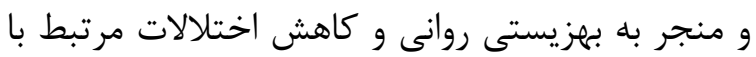

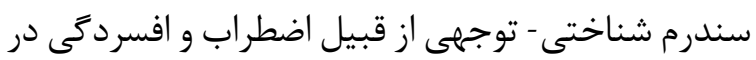

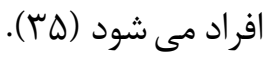

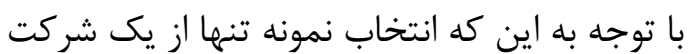

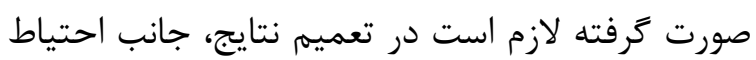

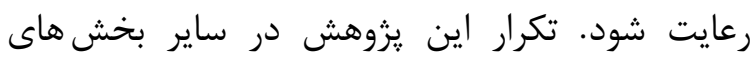

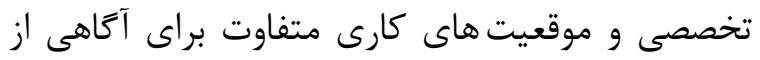


relations with mood and anxiety symptoms and distinctiveness from psychological inflexibility in a clinical sample. Psychiatry Res. 2013; 210(1):215-9.

24. Dickson KS, Ciesla JA, Reilly LC. Rumination, Worry, Cognitive Avoidance and Behavioral Avoidance: Examination of Temporal Effects. Behav Ther. 2012;43(3):629-40.

25. Fergus TA, Bardeen JR, Orcutt HK. Attentional Control Moderates the Relationship between Activation of the Cognitive Attentional Syndrome and Symptoms of Psychopathology. Personal Individ Diff. 2012;53(2):213217.

26. Mashhadi A, Mirardoghi F, Hasani J. The Role of Cognitive Emotional Ordering Strategies in Children's Internalization Disorders. J Behav Sci. 2011;3(3):39-29. [Persian]

27.Wells A, Welford M, King P, Papageorgiou C, Wisley J, Mendel E. A Pilot Randomized Trial of Metacognitive Therapy vs Applied Relaxation in the Treatment of Adults with Generalized Anxiety Disorder. Behav Res Ther. 2010;48(5):429-34.

28. Salmani B, Hasani J. Cognitive Attentional Syndrome (CAS) \& Cognitive Emotion Regulation Strategies: Transdiagnostic Processes or Diagnostic based on Mood \& Anxiety Disorders. J Clini Psychol. 2013;3(19):91-104. [Persian]

29. Steinmetz J. The stress reduction program at university hospital. San Diego:University of California Medical Center.1977.

30. Attar h. The Relationship between Job Stress and Job Satisfaction and Mental Health of an Industrial Complex. Master of Clinical Psychology, Tehran Psychiatric Institute. Iran University of Medical Sciences.1995. [Persian]

31. Hasanzade IOM, Bahrebardar MJ. Relationship between job stress and psychological health in Shiraz university staff. J Iran Psychi Psychol (think and beha). 2000;6(23)21-25. [Persian]

32. Sendi F, Ehteshamzadeh P, Asgary P, Kafie M. The relationship Cognitive Attentional Syndrome (CAS), with anxiety and depression in burned patients: the mediating role of coping styles. Health Psychology. 2017;3(6):74-91.

33. Gustems-Carnicer J, Calderón C. Coping strategies and psychological well-being among teacher education students Coping and well-being in students. Europ J Psychol Edu. 2012; 28(4):443-455.

34. Sanjuan P, Magallares A. Coping strategies as mediator variables between explanatory styles an depressive symptoms. anales de psicología. 2017;31:447-451.

35. Ra Y, Trusty J. Coping Strategies for Managing Acculturative Stress Among Asian International Students. Internati J Adva Counse. 2016; 37: 313-29. heartdiseases: Evidence and strategies for prevention. Geneva:International Labour Office. 1993;1-13.

9. Wells A. Metcognitive therapy for anxiety and depression, A practical guide. 5th Ed, New York, Guilford press. 2008;407-483.

10. Wells A, King P. Metacognitive Therapy for Generalized Anxiety Disorder: An Open Trial. J Behav Ther Exp Psychiatry. 2006;37(3):206-12.

11. Muris P, Roelofs J, Rassin E, Franken I, Mayer B. Mediating effects of rumination and worry on the links between neuroticism, anxiety and depression. Personal and Individ Differe. 2005;39:1105 -1111.

12. Segerstorm SC, Stanton AL, Alden LE, Shortridge BE. A multidimensional structure for repetitive thought: what's on your mind, and how, and how much? J Personal and Soc Psychol. 2003;85:909-921.

13. Brosschot JF. Markers of chronic stress: Prolonged physiological activation and (un) conscious perseverative cognition. Neur Behav Rev. 2010;35:46-50.

14. Watkins ER. Constructive and unconstructive repetitive thought. Psychol Bull. 2008;134(2): 163-206.

15. Ehring T, Watkins ER. Repetitive negative thinking as a transdiagnostic process. Internati J Cognit psychoth. 2008;1(3):192-205.

16. Nolen-Hoeksema S, Morrow J. A prospective study of depression and posttraumatic stress symptoms after a natural disaster: The 1989 Loma Prieta earthquake. J of Personal Soc Psychol.1991;61(2):115121-.

17. Borkovec TD, Ray WJ Stöber J. Worry: A cognitive phenomenon intimately linked to affective, physiological, and interpersonal behavioral processes. Cognit Ther and Res.1998; 22(6):561-576.

18. Rapee R, Heimberg RG. A cognitive-behavioral model of anxiety in social phobia. Behavio Res Ther. 1997;35:741756.

19. Brosschot JF, Gerin W, Thayer JF. The preservative cognition hypothesis: a review of worry, prolonged stress-related physiological activation, and Health. J Psycho Res. 2006;60, 113-124.

20. Verplanken B, Friborg O, Wang CE, Trafirmow D, Woolf K. Mental habits: metacognitive reflection on negative self-thinking. J Personal Soc Psychol. 2007;92:526-541.

21. Razmi S, Nemati Sogoli-Tapeh F. The moderating role of self- efficacy in relationship between occupational stress with psychological health and job satisfaction of Saderat Bank's Staffs of Tabriz. Iran Occupati Hea. 2011; 8:57-65. [Persian]

22. Yousefi Sh, Nayebzadeh Sh, Eslami H. The effects of job stress on Accountants job satisfaction. Iran Occupati Hea. 2015;12(3):46-53. [Persian]

23. Fergus TA, Valentiner DP, McGrath PB, Gier-Lonsway S, Jencius $S$. The cognitive attentional syndrome:Examining 